\title{
Forscher untersuchen Zusammenspiel von Chemie und Mechanik
}

\section{in Werkstoffen}

Wissenschaftler der Ruhr-Universität Bochum (RUB) untersuchen das Zusammenspiel von Chemie und Mechanik in technischen Werkstoffen in einem neu eingerichteten Schwerpunktprogramm der Deutschen Forschungsgemeinschaft (DFG). Mit Computermodellen wollen sie dazu beitragen, Produktionsprozesse, Gebrauchseigenschaften und die Lebenszeit von Werkstoffen zu optimieren. Im Zentrum des Projekts stehen Werkstoffe mit sogenannter thermo-chemo-mechanischer Kopplung. Beispiele für solche Materialien sind hochfeste Stähle oder Superlegierungen auf Nickel-Basis sowie faserverstärkte Polymere. Professor Dr. Ingo Steinbach vom Interdisciplinary Centre for Advanced Materials Simulation (ICAMS) der RUB koordiniert das Programm "Strong Coupling of Thermochemical and Thermo-mechanical States in Applied Materials", das die DFG zunächst für drei Jahre fördert. Etwa 20 Institute aus ganz Deutschland sind beteiligt.

"Metalle werden üblicherweise als tote Körper mit einer eingefrorenen inneren Struktur angesehen“, erläutert Steinbach. Aber das sei nicht so, denn ähnlich eines menschlichen Haars, das sich je nach Feuchtigkeit der Umgebung locke, reagierten auch Metalle, wenn sich die chemischen Bedingungen oder die mechanischen Belastungen ändern würden. Chemische Aspekte beein- flussten ihre mechanischen Eigenschaften, und eine mechanische Belastung wirke auf die Chemie zurück. Bislang hätten Forscher Chemie und Mechanik meist separat voneinander betrachtet. Im neuen Schwerpunktprogramm wollen die Wissenschaftler das wechselseitige Zusammenspiel dieser Aspekte in zwei grundlegend verschiedenen Materialklassen - Metallen und Polymeren analysieren. Die Erkenntnisse, gewonnen aus der Synergie der unterschiedlichen Materialklassen, sollen in neuartige Computermodelle münden. Zu diesem Zweck kooperieren experimentell und theoretisch arbeitende Wissenschaftler aus Materialwissenschaft, Kontinuumsmechanik und Thermodynamik. In der thermo-chemo-mechanischen Kopplung sind temperaturabhängige mechanische Prozesse stark mit temperaturabhängigen chemischen Prozessen verwoben. Die mechanischen Eigenschaften der unter- suchten Materialien seien abhängig von der sogenannten Phasenstruktur. Darunter werde die chemische Zusammensetzung, die Anordnung der Moleküle und die Verteilung verschiedener Phasen, die sich in Kristallstruktur oder Aggregatzustand unterscheiden, verstanden. „Diese Materialien kann man nicht verstehen, wenn man das Zusammenspiel der Phasenstruktur und Mechanik ignoriert", betont Steinbach.
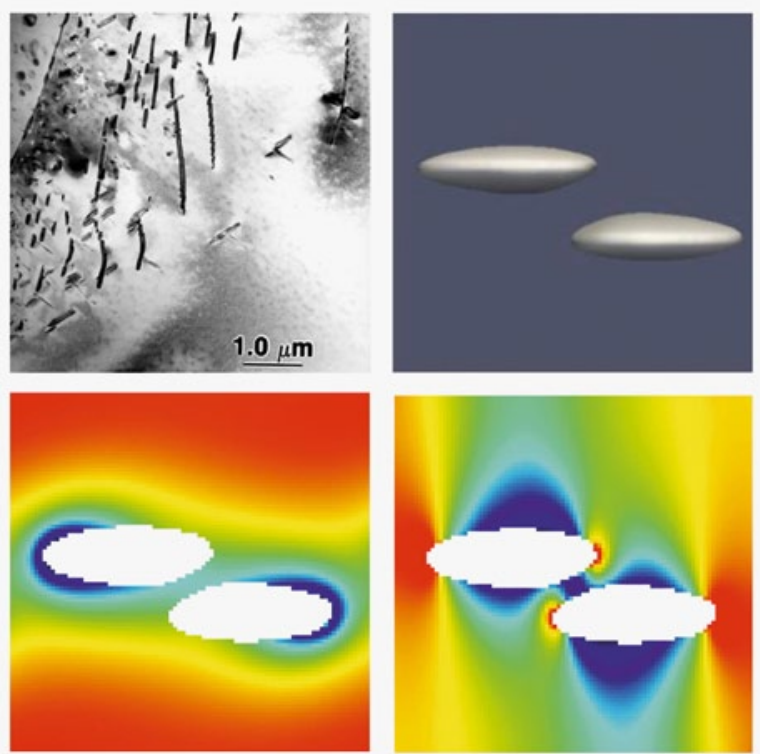